\title{
A Multi-Model Approach for System Diagnosis
}

\section{Niemann, Hans Henrik; Poulsen, Niels Kjølstad; Bækgaard, Mikkel Ask Buur}

\section{Published in:}

A Multi-Model Approach for System Diagnosis

Link to article, DOI:

10.1109/ACC.2007.4282628

Publication date:

2007

Document Version

Publisher's PDF, also known as Version of record

Link back to DTU Orbit

Citation (APA):

Niemann, H. H., Poulsen, N. K., \& Bækgaard, M. A. B. (2007). A Multi-Model Approach for System Diagnosis. In A Multi-Model Approach for System Diagnosis (pp. 2539-2544). Proceedings of the 2007 American Control Conference. https://doi.org/10.1109/ACC.2007.4282628

\section{General rights}

Copyright and moral rights for the publications made accessible in the public portal are retained by the authors and/or other copyright owners and it is a condition of accessing publications that users recognise and abide by the legal requirements associated with these rights.

- Users may download and print one copy of any publication from the public portal for the purpose of private study or research.

- You may not further distribute the material or use it for any profit-making activity or commercial gain

- You may freely distribute the URL identifying the publication in the public portal 


\section{A Multi-Model Approach for System Diagnosis}

\author{
Henrik Niemann \\ Ørsted•DTU, Automation \\ Tech. Univ. of Denmark \\ Build. 326, DK-2800 \\ Kgs. Lyngby, Denmark \\ hhndoersted.dtu.dk
}

\author{
Niels Kjølstad Poulsen \\ Informatics and Math. Modelling \\ Tech. Univ. of Denmark \\ Build. 321, DK-2800 \\ Kgs. Lyngby, Denmark \\ nkp@imm.dtu.dk
}

\author{
Mikkel Ask Buur Bækgaard \\ Ørsted•DTU, Automation \\ Tech. Univ. of Denmark \\ Build. 326, DK-2800 \\ Kgs. Lyngby, Denmark \\ mbbeaquarden. com
}

\begin{abstract}
A multi-model approach for system diagnosis is presented in this paper. The relation with fault diagnosis as well as performance validation is considered. The approach is based on testing a number of pre-described models and find which one is the best. It is based on an active approach, i.e. an auxiliary input to the system is applied. The multi-model approach is applied on a wind turbine system.
\end{abstract}

\section{INTRODUCTION}

The area of active fault diagnosis (AFD) has been considered in a number of books [3], [6], [16] and in papers [1], [2], [4], [13], [12], [14]. Further, in [9], an AFD approach has been considered for closed-loop systems. However, it has been shown in [10] that it is possible to apply the same AFD set-up on open-loop systems as well.

The AFD is based on the inclusion of an auxiliary input signal/vector into the system. The auxiliary input can either be injected in the open-loop system or in the closed-loop feedback system. As output from the system a standard residual vector known from the passive FD (fault diagnosis) approach is applied [5]. Using the AFD approach from [9], [8], the auxiliary input is injected in the closed-loop system in such a way that the standard residual vector is decoupled from this auxiliary input in the nominal case and coupled in the faulty case. It turns out that an optimal placement of the auxiliary input vector together with the selection of the standard residual vector as the output vector connect the AFD with the dual YJBK (after Youla, Jabr, Bongiorno and Kucera) parameterization, [7], [15]. The transfer function from auxiliary input to the residual vector is equivalent to the dual YJBK transfer function in the dual YJBK parameterization, i.e. a parameterization of all systems stabilized by a given feedback controller. Here, in connection with AFD, this transfer function has been named the fault signature matrix, [9], [8]. Fault detection as well as fault isolation are based directly on the fault signature matrix.

There are mainly two approaches in AFD. In the approach derived by Campbell et.al., [3], the auxiliary input is designed with respect to a fast fault diagnosis/multi-model selection. Using a dedicated design of the auxiliary input gives a fast diagnosis/selection.

The other AFD approach in [8], [10] and applied in [11], a periodic auxiliary input is applied. The signature from this periodic input in the output/residual vector is quite distinct and will also be a periodic vector with the same frequency. A change in the system due to e.g. parametric faults, will give a change in the signature. This change can be in the amplitude and/or the phase of the periodic output vector.

Using the AFD approach from [8], [10], the auxiliary input is decoupled in the output/residual vector in the nominal case. The detection of parametric faults can then be done by a detection of a signature from the auxiliary input in the residual vector. Another approach is to use a filter/observer to estimate the periodic signature with the known frequency directly. This approach will not be considered in this paper.

Instead of using the AFD approach applied in [10], [11], where the residual vector is derived by using the nominal model, it is possible to use a number of models in connection with the fault diagnosis. This approach has been applied in e.g. [1]. The main issue in this paper is to apply a multimodel approach in connection with the AFD set-up derived in [10], [11]. The direct consequence of this change is that instead of considering a single residual vector based on the nominal model, a number of residual vectors are calculated based on different models. The system changes can then be identified by a detection in which residual vector the signature from the auxiliary is smallest/minimal. It will be shown in this paper that ability to identify system changes will not depend directly on the number of models applied. By using information from more than a single residual vector it is possible to get a very good diagnosis based on a few models.

The multi-model AFD approach described in this paper is applied on a wind turbine system. The wind turbine system is non-linear. This non-linearity will allow to detect changes in the working point based on changes in the system dynamic. In this case it will be possible to estimate the wind speed based on a detection of changes in the dynamic in the wind turbine.

\section{SYSTEM SET-UP}

Let a general system be given by:

$$
\Sigma:\left\{\begin{array}{l}
z=G_{z w} w+G_{z d} d+G_{z u} u \\
e=G_{e w} w+G_{e d} d+G_{e u} u \\
y=G_{y w} w+G_{y d} d+G_{y u} u
\end{array}\right.
$$


where $d \in \mathcal{R}^{r}$ is a disturbance signal vector, $u \in \mathbb{R}^{m}$ the control input signal vector, $e \in \mathcal{R}^{q}$ is the external output signal vector to be controlled, $y \in \mathcal{R}^{p}$ is the measurement vector, $w \in \mathcal{R}^{k}$ and $z \in \mathcal{R}^{k}$ are external input and output vectors. The connection between the external output and the external input is given by

$$
w=\theta z
$$

where $\theta$ is a diagonal matrix given by

$$
\theta=\operatorname{diag}\left(\theta_{1}, \cdots, \theta_{i}, \cdots, \theta_{k}\right)
$$

represents the system parameters. It is assumed that only these parameters can change in the system, the others are assumed to be constant. Closing the loop from $w$ to $z$ in $\Sigma$ by $\theta$ described by a linear fractional transformation (LFT) we get

$$
\Sigma_{\theta}=\mathcal{F}_{u}(\Sigma, \theta),
$$

where $\Sigma_{\theta}$ is given by:

$$
\Sigma_{\theta}:\left\{\begin{array}{l}
e=G_{e d}(\theta) d+G_{e u}(\theta) u \\
y=G_{y d}(\theta) d+G_{y u}(\theta) u .
\end{array}\right.
$$

Further, let the system be controlled by a stabilizing feedback controller given by:

$$
\Sigma_{C}:\{u=K y .
$$

Let a coprime factorization of the system $G_{y u}$ from (1) and the stabilizing controller $K$ from (3) be given by:

$$
\begin{array}{ll}
G_{y u}=N_{0} M_{0}^{-1}=\tilde{M}_{0}^{-1} \tilde{N}_{0}, \quad N_{0}, M_{0}, \tilde{N}_{0}, \tilde{M}_{0} \in \mathcal{R} \mathcal{H}_{\infty} \\
K \quad=U_{0} V_{0}^{-1}=\tilde{V}_{0}^{-1} \tilde{U}_{0}, \quad U_{0}, V_{0}, \tilde{U}_{0}, \tilde{V}_{0} \in \mathcal{R} \mathcal{H}_{\infty},
\end{array}
$$

where the eight matrices in (4) must satisfy the double Bezout equation given by, [15]:

$$
\begin{aligned}
\left(\begin{array}{ll}
I & 0 \\
0 & I
\end{array}\right) & =\left(\begin{array}{cc}
\tilde{V}_{0} & -\tilde{U}_{0} \\
-\tilde{N}_{0} & \tilde{M}_{0}
\end{array}\right)\left(\begin{array}{cc}
M_{0} & U_{0} \\
N_{0} & V_{0}
\end{array}\right) \\
& =\left(\begin{array}{cc}
M_{0} & U_{0} \\
N_{0} & V_{0}
\end{array}\right)\left(\begin{array}{cc}
\tilde{V}_{0} & -\tilde{U}_{0} \\
-\tilde{N}_{0} & \tilde{M}_{0}
\end{array}\right) .
\end{aligned}
$$

\section{A. The YJBK Parameterization}

Based on the above coprime factorization of the system $G_{y u}$ and the controller $K$ we can give a parameterization of all controllers that stabilize the system in terms of a stable transfer function $Q$, i.e. all stabilizing controllers are given by [15]:

$$
K(Q)=\left(U_{0}+M_{0} Q\right)\left(V_{0}+N_{0} Q\right)^{-1}, Q \in \mathcal{R} \mathcal{H}_{\infty},
$$

or by using a left factored form:

$$
K(Q)=\left(\tilde{V}_{0}+Q \tilde{N}_{0}\right)^{-1}\left(\tilde{U}_{0}+Q \tilde{M}_{0}\right), Q \in \mathcal{R} \mathcal{H}_{\infty} .
$$

Using the Bezout equation the controller given either by (6) or by (7) can be realized as an LFT in $Q$ :

$$
K(Q)=\mathcal{F}_{l}\left(\left(\begin{array}{cc}
U_{0} V_{0}^{-1} & \tilde{V}_{0}^{-1} \\
V_{0}^{-1} & -V_{0}^{-1} N_{0}
\end{array}\right), Q\right)=\mathcal{F}_{l}\left(J_{K}, Q\right) .
$$

\section{B. The Dual YJBK Parameterization}

In the same way it is possible to derive a parameterization in terms of a stable transfer function $S$ of all systems that are stabilized by one controller, i.e. the dual YJBK parameterization. The parameterization is given by [15]:

$$
G_{y u}(S)=\left(N_{0}+V_{0} S\right)\left(M_{0}+U_{0} S\right)^{-1}, S \in \mathcal{R} \mathcal{H}_{\infty}
$$

or by using a left factored form:

$$
G_{y u}(S)=\left(\tilde{M}_{0}+S \tilde{U}_{0}\right)^{-1}\left(\tilde{N}_{0}+S \tilde{V}_{0}\right), S \in \mathcal{R} \mathcal{H}_{\infty} .
$$

An LFT representation of (9) or (10) is given by:

$$
G_{y u}(S)=\mathcal{F}_{l}\left(\left(\begin{array}{cc}
N_{0} M_{0}^{-1} & \tilde{M}_{0}^{-1} \\
M_{0}^{-1} & -M_{0}^{-1} U_{0}
\end{array}\right), S\right)=\mathcal{F}_{l}\left(J_{G}, S\right) .
$$

Further $S$ is given by, [15]:

$$
S=\mathcal{F}_{u}\left(J_{K}, G_{y u}(S)\right)
$$

\section{AFD Set-up}

Now, let's also including a residual vector in connection with the system.

It has been shown in [5] that it is possible to parameterize all residual generators by using the YJBK parameterization. All residual vectors $\varepsilon_{0}$ for $\Sigma$ given by (1) can be described by:

$$
\varepsilon_{0}=\tilde{M}_{0} y-\tilde{N}_{0} u \text {. }
$$

Let's consider the block diagram shown in Fig. 1. The diagram includes the residual vector $\varepsilon_{0}$ and an auxiliary input vector $\eta$. The two signal vectors, $\eta$ and $\varepsilon_{0}$, will be applied in connection with the AFD, see [8], [11].

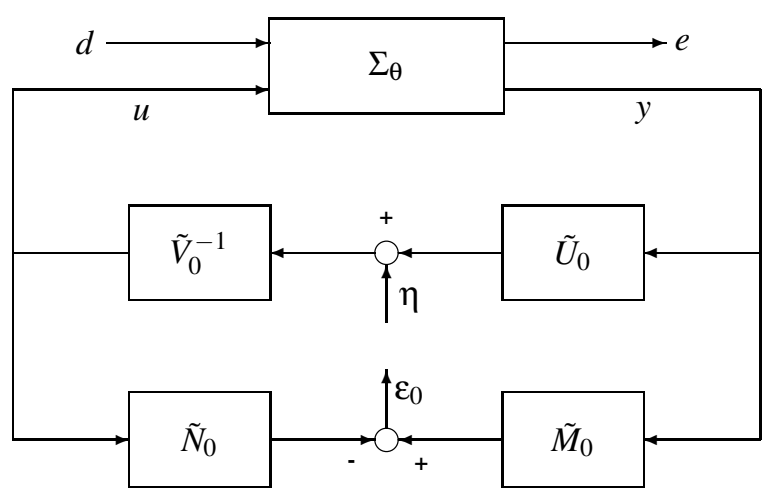

Fig. 1. Controller structure including residual vector $\varepsilon_{0}$ and the external input vector $\eta$.

Based on the feedback system in Fig. 1 the transfer functions from the two input vectors $d, \eta$ to the two output vectors $e, \varepsilon_{0}$ are given by:

$$
\Sigma_{\mathrm{FD}}:\left\{\begin{array}{c}
e=P_{e d}(\theta) d+P_{e \eta}(\theta) \eta \\
\varepsilon_{0}=P_{\varepsilon d}(\theta) d+P_{\varepsilon \eta}(\theta) \eta,
\end{array}\right.
$$

where the explicit equations for $\Sigma_{\mathrm{FD}}$ can be found in [8], [11].

Note that the transfer function from the input vector $\eta$ to the residual vector $\varepsilon_{0}$ is equal to the dual YJBK transfer 
function, [8], [11]. The explicit equation for $S=P_{\varepsilon \eta}$ is given by, [7]:

$$
S(\theta)=\tilde{M}_{0} G_{y w} \theta\left(I-\left(G_{z w}+G_{z u} U_{0} \tilde{M}_{0} G_{y w}\right) \theta\right)^{-1} G_{z u} M_{0} .
$$

(15) gives a direct description of the parameter variations' effect on the closed-loop stability. If $S$ gets unstable for some parameter variations, the closed-loop system will be unstable. A large $S$ indicate that the parameter variations has a major effect on the system. In [10], [11], $S(\theta)$ was named as the fault signature matrix.

Based on the fault signature matrix $S(\theta)$ the conditions for fault detection are as follows, [10], [11]:

- Fault detection

$$
\begin{aligned}
& S(\theta)=0, \quad \text { for } \theta=\bar{\theta} \\
& S(\theta) \neq 0, \quad \text { for } \theta \neq \bar{\theta} .
\end{aligned}
$$

\section{Multi-Model AFD Set-UP}

It is possible to extend the AFD set-up shown in Fig. 1 to a multi-model AFD, MM-AFD, set-up. This can be done by including a number of residual vectors that are derived based on different models. This is shown in Fig 2.

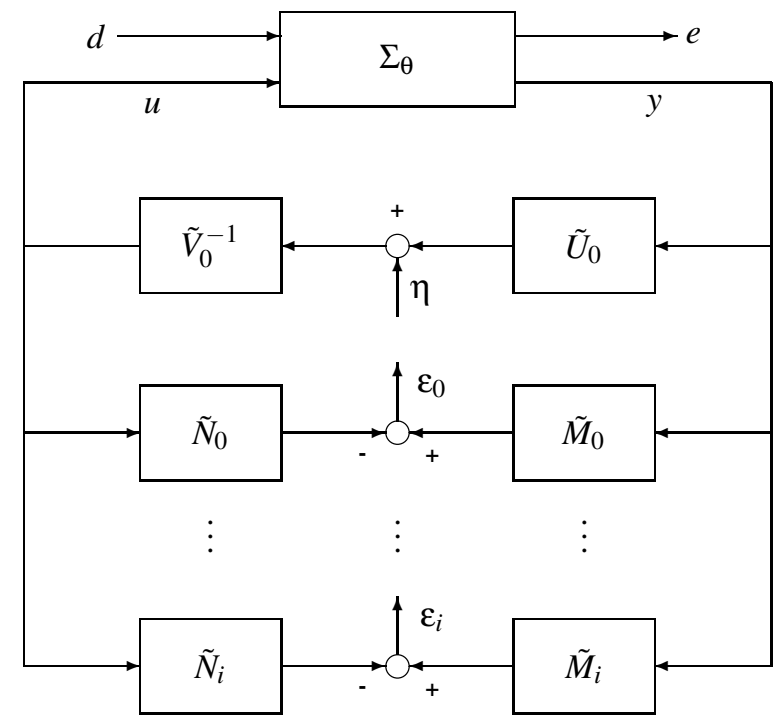

Fig. 2. The AFD set-up based on a multi model approach. $\left(\tilde{M}_{i}, \tilde{N}_{i}\right)$ is the $i^{\prime}$ th model with $i=0$ is the nominal model.

Let the transfer function from $\eta$ to the residual vector $\varepsilon_{i}$ be given by $S_{i}(\theta)$. This will give the following simple conditions for system model identification for a given $\bar{\theta}$ :

- System model identification

$$
\begin{aligned}
& S_{i}(\bar{\theta})=0, \text { for } \bar{\theta}=\bar{\theta}_{i} \\
& S_{j}(\bar{\theta}) \neq 0, \text { for } \bar{\theta} \neq \bar{\theta}_{i}, i \neq j .
\end{aligned}
$$

It is clear that in general it is not possible to require that one of the fault signature matrices, $S_{i}(\theta)$, is exact equal to zero for the identification of the correct model. The reason is that only a limited number of models will be applied and it will therefore be very difficult to require that one of the fault signature matrices is exact zero.
Instead of just using different models for the calculation of different residual vectors we can again use the dual YJBK parameterization. Let the model be parameterized by $\bar{S}_{i}$, the residual vector given by (13) then takes the following form:

$$
\varepsilon_{i}=\left(\tilde{M}_{0}+\bar{S}_{i} \tilde{U}_{0}\right) y-\left(\tilde{N}_{0}+\bar{S}_{i} \tilde{V}_{0}\right) u, \bar{S}_{i} \in \mathcal{R} \mathcal{H}_{\infty} .
$$

Using the dual YJBK parameterization of $G_{y u}$ given by (9) or (10), the transfer functions from $\eta$ to $y$ and $u$ are given by:

$$
\begin{aligned}
& y=\left(N_{0}+V_{0} S\right) \eta+V_{0}\left(\tilde{M}_{0}+S \tilde{U}_{0}\right) G_{y d}(\theta) d \\
& u=\left(M_{0}+U_{0} S\right) \eta+\left(M_{0}+U_{0} S\right) \tilde{U}_{0} G_{y d}(\theta) d .
\end{aligned}
$$

Together with the residual vector in (16) gives the following residual vector:

$$
\begin{aligned}
\varepsilon_{i} & =\left(S-\bar{S}_{i}\right) \eta+\left(\tilde{M}_{0}+S \tilde{U}_{0}\right) G_{y d}(\theta) d \\
& =\varepsilon_{0}-\bar{S}_{i} \eta .
\end{aligned}
$$

From (17) it is clear that if we select the correct model in the residual generator in terms of a fault signature matrix $\bar{S}_{i}$, then the residual vector will be independent of the system changes.

Let's apply a periodic auxiliary input in connection with the MM-AFD in the same line as described in [10], [11] for AFD. The reason for using a periodic auxiliary input is that it is easy to detect the signature from $\eta$ in the residual vector $\varepsilon_{i}$. Let the auxiliary input $\eta$ be given by:

$$
\eta=A_{\eta} \sin \left(\omega_{0} t\right)
$$

where the amplitude $A_{\eta}$ and the frequency $\omega_{0}$ are design parameters. These parameters need to be selected in such a way that the effect from $\eta$ is maximized in $\varepsilon_{i}$ and minimized in $e$, see [11] for further details. The residual vector based on the $i^{\prime} t h$ model is:

$$
\varepsilon_{i}=A_{\eta}\left|S(\theta)-\bar{S}_{i}\right| \sin \left(\omega_{0} t+\phi\right)+P_{\varepsilon d}(\theta, i) d .
$$

Consider the residual $\varepsilon_{i}$ at the specific frequency $\omega_{0}$, i.e.:

$$
\delta_{i}=\varepsilon_{i} \sin \left(\omega_{0} t+\phi\right)
$$

$\delta_{i}$ is then given by:

$$
\delta_{i}=A_{\eta}\left|S(\theta)-\bar{S}_{i}\right| \sin ^{2}\left(\omega_{0} t+\phi\right)+P_{\varepsilon d}(\theta, i) d \sin \left(\omega_{0} t+\phi\right) .
$$

Further, in the Gaussian case $\delta_{i}$ will satisfy:

$$
\delta_{i} \in \mathbf{N}\left(\left|S(\theta)-\bar{S}_{i}\right| A_{\eta} \sin ^{2}\left(\omega_{0} t+\phi\right), \sigma^{2} \sin ^{2}\left(\omega_{0} t+\phi\right)\right),
$$

where $\sigma^{2}$ is the variance of the signature from the disturbances in the residual. In (time) average we have the following mean and variance:

$$
\bar{\mu}=\frac{1}{2}\left|S(\theta)-\bar{S}_{i}\right| A_{\eta} \quad \bar{\sigma}^{2}=\frac{1}{2} \sigma^{2} .
$$

Assume that the frequency $\omega_{0}$ has been selected in such a way that the amplitude of $\varepsilon_{i}$ given by $\left|S(\theta)-\bar{S}_{i}\right| A_{\eta}$ is a monotonic increasing function of $|\bar{\theta}-\theta|$. Local minima in the fault signature matrix might occur. This will not give a monotonic increasing amplitude as function of $|\bar{\theta}-\theta|$. This can be avoided in different ways, e.g. by selecting 
another $\omega_{0}$, changing the models or incorporating it in the identification of the system model.

Assume that a number of models described by $\bar{\theta}_{i}, i \in N$ are considered. Assume that $\left|S\left(\theta, \omega_{0}\right)-\bar{S}_{i}\left(\omega_{0}\right)\right|$ are increasing functions of $\left|\bar{\theta}_{i}-\theta\right|$. Let the real system be described by $\theta \in\left[\bar{\theta}_{i}, \bar{\theta}_{i+1}\right]$. Then we will have:

$$
\begin{aligned}
A_{\eta}\left|S(\theta)-\bar{S}_{i}\right| & <A_{\eta}\left|S(\theta)-\bar{S}_{i-1}\right|<\cdots \\
A_{\eta}\left|S(\theta)-\bar{S}_{i+1}\right| & <A_{\eta}\left|S(\theta)-\bar{S}_{i+2}\right|<\cdots
\end{aligned}
$$

for $\omega=\omega_{0}$. See e.g. Fig. 4 , where the gains of the fault signature matrices are shown for a wind turbine system.

By using interpolation it is possible to identify a very precise model based on a limit number of pre-specific models.

Normally, only the models close to the real system are considered in connection with identification of the real system. However, it is also relevant to use the residual vectors based on the other models. The reason is that the signal-noise ratio in general will be much better than residuals based on models close to the real system.

The focus in the above has been on identification of system models due to change in parameters. It has also been assumed that the systems are linear, but it might not be linear. If the system is non-linear, it is still possible to use the MMAFD set-up. The fault signature matrix will be a function of the difference between the nominal system and the real non-linear system. In general it will not be possible to give an explicit equation for $S$ as in the linear case. $S$ can be considered as a measure of the change in the dynamic cause by changes of the working point. This mean that $S$ can be applied as an indirect measure of the working point for nonlinear systems. The MM-AFD set-up used on a non-linear system is shown below, where a wind turbine system is considered. The variation in the working point is caused by changes in the wind speed. Using the MM-AFD approach on the wind turbine it is shown that it is possible to estimate the wind speed on the system quite well.

\section{EXAMPLE}

The example is a $2 \mathrm{MW}$ wind turbine with a three blade rotor. Since the model includes models for the actuators the wind turbine can be described by a 5th order non-linear model given by:

$$
\begin{aligned}
& \dot{x}=f(x, u, d) \\
& y=g(x, u, d),
\end{aligned}
$$

where the 5 states $x=\left[\omega_{r}, \omega_{g}, \delta, T_{g}, \theta_{b}\right]^{T}$ are the rotor speed, the generator speed, the deformation of the drive train, the generator torque, and the pitch angle, respectively, the control inputs $u=\left[T_{g, r e f}, \theta_{b, r e f}\right]^{T}$ are the reference to the generator torque and the pitch angle, and the disturbance $d=v_{r}$ is the wind speed. The applied model describes the wind turbine in the top region, for wind speed from 11 to $26 \mathrm{~m} / \mathrm{sec}$. A linear model is derived for a wind speed around $18 \mathrm{~m} / \mathrm{sec}$. The state space matrices are given in Appendix.

The wind speed is modeled as a second order system driven by white noise. The gain and the poles depend on the nominal wind speed.
The system is extended with two integrators to remove steady state errors in connection with the control of the wind turbine. The extended linear model is given by:

$$
\begin{aligned}
\left(\begin{array}{c}
\dot{x} \\
\dot{x}_{\text {int }}
\end{array}\right)= & \left(\begin{array}{cc}
A & 0 \\
-C_{e} & 0
\end{array}\right)\left(\begin{array}{c}
x \\
x_{\text {int }}
\end{array}\right)+\left(\begin{array}{c}
B_{d} \\
0
\end{array}\right) d \\
& +\left(\begin{array}{c}
B_{u} \\
0
\end{array}\right) u+\left(\begin{array}{c}
0 \\
I
\end{array}\right) r_{\text {ref }} \\
e= & \left(\begin{array}{cc}
C_{e} & 0
\end{array}\right)\left(\begin{array}{c}
x \\
x_{i n t}
\end{array}\right) \\
y= & I\left(\begin{array}{c}
x \\
x_{\text {int }}
\end{array}\right) .
\end{aligned}
$$

An LQR state feedback controller is designed for the extended system. The controller gain is given in Appendix.

The MM-AFD set-up for the wind turbine will be based on three models specified at the following wind speeds: $13 \mathrm{~m} / \mathrm{sec}$., $18 \mathrm{~m} / \mathrm{sec}$. and $23 \mathrm{~m} / \mathrm{sec}$. Based on these three models three Kalman filters are designed with respect to the disturbance in the system. Using a Kalman filter, the residual vector is given directly as the innovation vector from the filter.

Based on the feedback controller and the Kalman filter based on the model at $18 \mathrm{~m} / \mathrm{sec}$, an analysis of the fault signature matrix is derived. The analysis is not simple because it is possible to apply 2 inputs and 5 outputs. This results in a fault signature matrix of dim. $2 \times 5$. The maximal singular value of $S$ as function of change in the wind speed and the frequency is shown in Fig. 3.

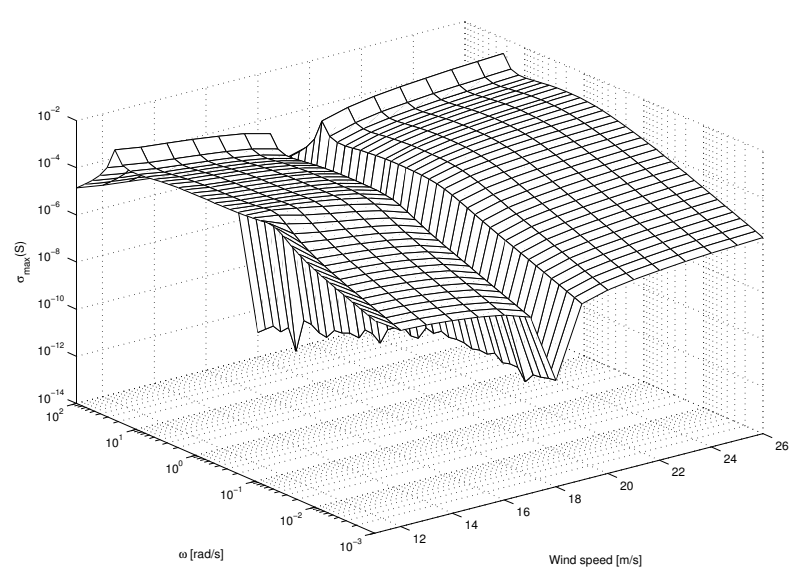

Fig. 3. The maximal singular value of $S$ as function of change in the wind speed and the frequency. $18 \mathrm{~m} / \mathrm{sec}$ is the nominal wind speed.

The selection of the frequency for the periodic auxiliary input signal is quite complicated in this case. From Fig. 3, it turns out that an obvious choice is to select $\omega_{0}$ either at low frequencies or at frequencies around $10 \mathrm{rad} / \mathrm{sec}$. Considering the ratio $\left(S / P_{e \eta}\right)$ gives that the frequency should be selected at high frequencies. However, also the signal to noise ratio as well as the dynamic in the system need to be considered in connection with the selection of the frequency. This includes 
that there are bounds on how fast it is possible to activate the wind turbine. With respect to these constraints the periodic auxiliary input is selected with $\omega_{0}=2.0 \mathrm{rad} / \mathrm{sec}$.

The next thing to consider is the input-output directions of the fault signature matrix that need to be applied to get the best residual output. An analysis shows that the optimal input/output directions are to use the second input $\theta_{b, \text { ref }}$ and the second output $\omega_{g}$.

The selection of the frequency and the directions has only been based on the Kalman filter for the model with respect to a wind speed at $18 \mathrm{~m} / \mathrm{sec}$. These selections have been verified with respect to the other two Kalman filters.

Based on the three residual signals (vectors) from the three Kalman filters the gain of the $S_{i}$ as function of variations in the wind speed is calculated for the selected frequency $\omega_{0}=2.0 \mathrm{rad} / \mathrm{sec}$. In Fig. 4 the gains of the transfer function from $\eta$ to $\varepsilon$ for the three fault signature matrices are shown.

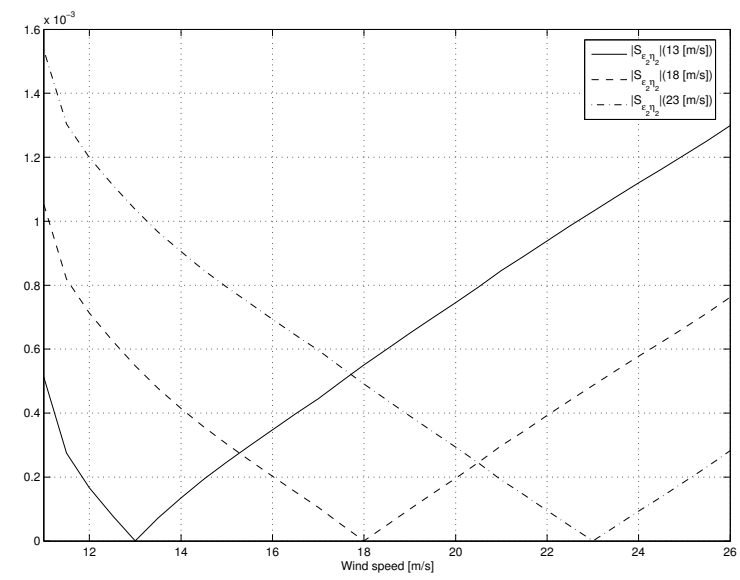

Fig. 4. The gain of the transfer function from $\eta$ to $\varepsilon$ for the three fault signature matrices at $\omega_{0}=2.0 \mathrm{rad} / \mathrm{sec}$.

The amplitude of the auxiliary input signal is selected to be 0.15 , i.e.

$$
\eta=0.15 \sin (2.0 t)
$$

The wind speed acting on the wind turbine is shown in Fig. 5. Note that there is a step in the mean wind speed at $300 \mathrm{sec}$. from $16 \mathrm{~m} / \mathrm{sec}$. to $18 \mathrm{~m} / \mathrm{sec}$. This wind speed will be used in the rest of the simulations.

Now let's consider the residual signals from the three Kalman filters. These residual signals are shown in Fig. 6, where a band-pass filter has been applied on the residuals.

As it can be seen from the residual signals it is almost impossible to see the signature from the auxiliary input signal. It is therefore not possible to use the residual signals directly. Instead of using the residual signals directly, we need only to consider the residual signals at the same frequency as used in the auxiliary input signal, i.e. $\omega_{0}=2.0 \mathrm{rad} / \mathrm{sec}$ as shown in (20). Using this new residual signal $\delta_{i}$ has a mean value that depends on the gain of $S_{i}$ and the amplitude of auxiliary input signal, see (23). It is then simple to estimate the mean

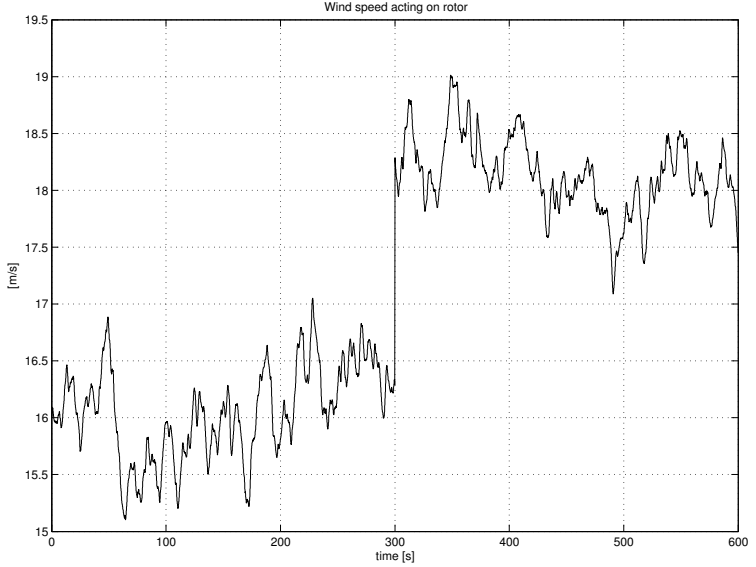

Fig. 5. The wind speed acting on the wind turbine.
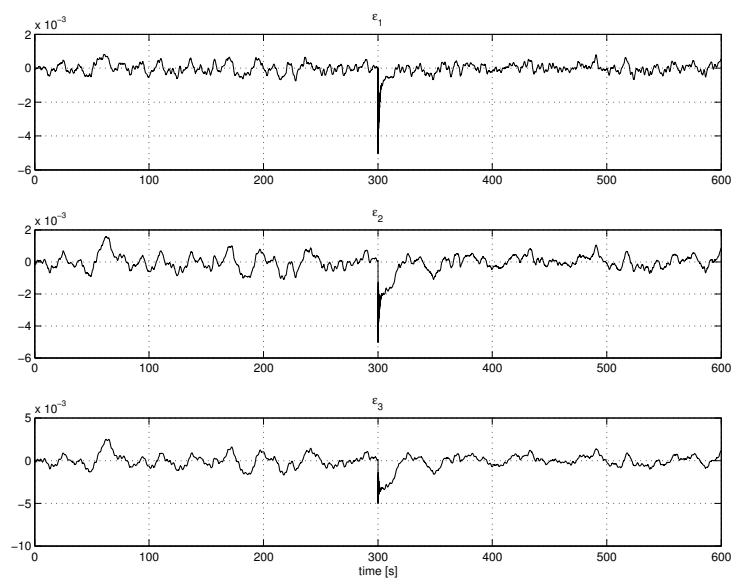

Fig. 6. The three residual signals.

value of $\delta_{i}$ by using an integration of the signal. This gives the following outputs:

$$
\kappa_{i}=\mid \int_{0}^{T}\left(\varepsilon_{i} e^{\left.j \omega_{0} t\right)} d t \mid, i=\{13,18,23\} .\right.
$$

In Fig. 7 the curves for the three $\kappa$ 's are shown. It is clear from the three curves that in the interval from $t=0 \mathrm{sec}$. to $300 \mathrm{sec}$., all three curves are constantly increasing. Further, it is also clear that the curve based on a wind speed at $23 \mathrm{~m} / \mathrm{sec}$. has the largest increasing rate. For $t=[0,300] \mathrm{sec}$. the three curves satisfy:

$$
\kappa_{18}<\kappa_{13}<\kappa_{23} .
$$

Combining this with the curves in Fig. 4 we get that the wind speed will be in between $13 \mathrm{~m} / \mathrm{sec}$. and $18 \mathrm{~m} / \mathrm{sec}$, which is also correct. For the time interval from $300 \mathrm{sec}$. to $600 \mathrm{sec}$, where the wind speed is changed from $16 \mathrm{~m} / \mathrm{sec}$. to $18 \mathrm{~m} / \mathrm{sec}$., we can see that the curve for $\kappa_{18}$ almost gets constant. This is in line with the associated curve in Fig. 4, where the gain of $S_{18}$ is zero for this wind speed. This indicates that the real wind speed is close to $18 \mathrm{~m} / \mathrm{sec}$. 


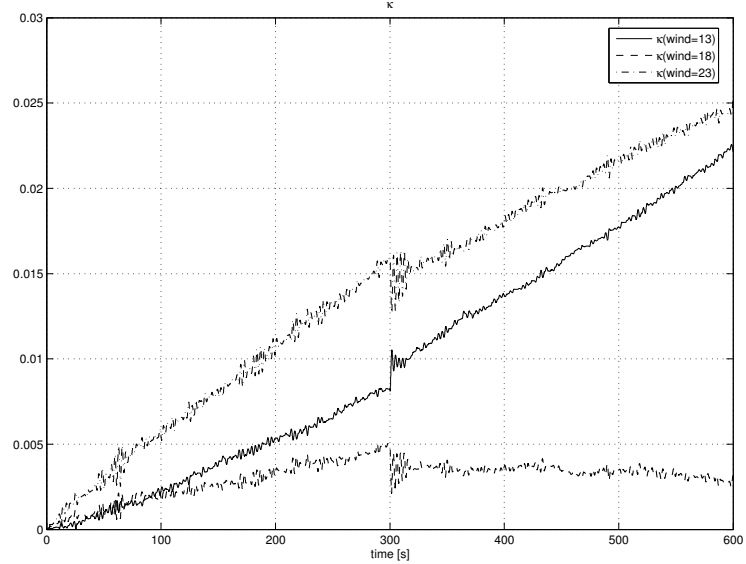

Fig. 7. The three $\kappa$ curves. The upper curve is for $\kappa_{23}$, the middle is for $\kappa_{13}$ and the lover one is for $\kappa_{18}$.

It is also possible to get an estimate of the wind speed by using the three curves in Fig. 7. This can be done in different ways. Here, the gains of the three fault signature matrices are estimated from the curves in Fig. 7.

Based on the estimates of the three gains the wind speed is then calculated by interpolation using the curves in Fig. 4. The estimated wind speed is shown in Fig. 8 together with the real wind speed. As it can be seen from Fig. 8 the estimated wind speed is reasonably good, except in the beginning.

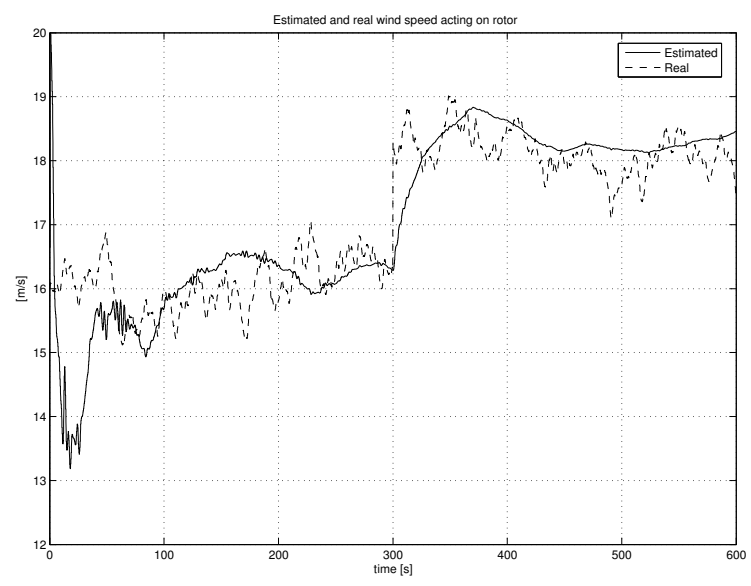

Fig. 8. Estimate of the wind speed together with the real wind speed.

\section{APPENDIX}

The state space matrices for a linear model of the wind turbine at $v_{r}=18 \mathrm{~m} / \mathrm{sec}$ are given by:

$A=\left[\begin{array}{ccccc}-0.37029 & 0.001672 & -14.212 & 0 & -0.027949 \\ 96.978 & -1.1409 & 9697.8 & -0.0066667 & 0 \\ 1 & -0.011765 & 0 & 0 & 0 \\ 0 & 0 & 0 & -10 & 0 \\ 0 & 0 & 0 & 0 & -5\end{array}\right]$

$$
\begin{aligned}
B_{d} & =\left[\begin{array}{c}
0.037945 \\
0 \\
0 \\
0 \\
0
\end{array}\right], B_{u}=\left[\begin{array}{cc}
0 & 0 \\
0 & 0 \\
0 & 0 \\
10 & 0 \\
0 & 5
\end{array}\right] \\
C_{e} & =\left[\begin{array}{ccccc}
0 & 11937 & 0 & 167.55 & 0 \\
1 & 0 & 0 & 0 & 0
\end{array}\right] .
\end{aligned}
$$

The LQR controller gain is given by:

$$
F_{L Q R}=\left[\begin{array}{ll}
F_{l q r} & F_{l q r, i}
\end{array}\right]
$$

where

$$
\begin{gathered}
F_{l q r}=\left[\begin{array}{ccccc}
-6082.3 & -8.6773 & 5468.3 & 0.093325 & 35.654 \\
-5.7077 & -0.0083656 & -0.26201 & 3.1279 e-007 & 0.0314
\end{array}\right] \\
F_{l q r, i}=\left[\begin{array}{cc}
-0.0054848 & 4775.4 \\
3.1172 e-007 & 1.4743
\end{array}\right] .
\end{gathered}
$$

\section{REFERENCES}

[1] S.L. Campbell, K.G. Horton, and R. Nikoukhah. Auxiliary signal design for rapid multi-model identification using optimization. Automatica, 38:1313-1325, 2002.

[2] S.L. Campbell, K.G. Horton, R. Nikoukhah, and F. Delebecque. Rapid model selection and the separability index. In Proceedings of Safeprocess 2000, pages 1187-1192, Budapest, Hungary, 2000.

[3] S.L. Campbell and R. Nikoukhah. Auxiliary signal design for failure detection. Princeton University Press, Princeton, NJ, USA, 2004.

[4] S.L. Campbell and R. Nikoukhah. Software for auxiliary signal design. In Proceedings of the American Control Conference, pages 44144419, Boston, MA, USA, 2004.

[5] P.M. Frank and X. Ding. Frequency domain approach to optimally robust residual generation and evaluation for model-based fault diagnosis. Automatica, 30:789-804, 1994.

[6] F. Kerestecioglu. Change detection and input design in dynamic systems. Research Studies Press, Baldock, Hertfordshire, 1993.

[7] H.H. Niemann. Dual Youla parameterization. IEE Proceedings Control Theory and Applications, 150(5):493-497, 2003.

[8] H.H. Niemann. Fault tolerant control based on active fault diagnosis. In Proceedings of the American Control Conference, pages 22242229, Portland, Oregon, USA, 2005.

[9] H.H. Niemann. Active fault diagnosis in closed-loop uncertain systems. In Preprints of 6th IFAC Symposium on Fault Detection Supervision and Safety for Technical Processes, SAFEPROCESS'2006, pages 631-636, Beijing, China, 2006.

[10] H.H. Niemann. A setup for active fault diagnosis. IEEE Transactions on Automatic Control, 51(9):1572-1578, 2006.

[11] H.H. Niemann and N.K. Poulsen. Active fault diagnosis in closed-loop systems. In Proceedings of the 16th IFAC World Congress, page 6, Prague, Czech Republic, 2005.

[12] R. Nikoukhah. Innovations generation in the presence of unknown inputs: Application to robust failure detection. Automatica, 30:18511867, 1994.

[13] R. Nikoukhah. Guaranteed active failure detection and isolation for linear dynamical systems. Automatica, 34(11):1345-1358, 1998.

[14] R. Nikoukhah, S.L. Campbell, and F. Delebecque. Detection signal design for failure detection: a robust approach. International Journal of Adaptive Control and Signal Processing, 14:701-724, 2000.

[15] T.T. Tay, I.M.Y. Mareels, and J.B. Moore. High performance control. Birkhäuser, 1997.

[16] X.J. Zhang. Auxiliary signal design in fault detection and diagnosis. Springer Verlag, Heidelberg, 1989. 